\title{
LES ESPÈCES ANIMALES INTRODUITES DANS LES EAUX DOUCES DE CORSE.
}

\author{
B. ROCHE (1), J. MATTEI (2).
}

(1) Direction Régionale de l'Environnement de la Corse, Service de l'Eau et des Milieux Aquatiques, B.P. 605, 20601 Bastia Cedex 1, France.

(2) Conseil Supérieur de la Pêche, Fédération Interdépartementale pour la Pêche et la Protection des Milieux Aquatiques, 7 boulevard Paoli, 20200 Bastia, France.

\begin{abstract}
RÉSUMÉ
Dix-sept espèces étrangères à la faune insulaire ont été introduites en moins d'un siècle dans les eaux douces, dont quinze espèces de poissons. Toutes se sont acclimatées dans différents types de milieux et contribuent à enrichir la biodiversité du peuplement aquatique et plus particulièrement l'ichtyocénose des lacs naturels ou artificiels. L'impact sanitaire, peu étudiè, est abordé avec mention de l'apparition récente de l'anguillicolose et l'impact génétique est traité à partir des connaissances récentes acquises sur les populations sauvages de truites.
\end{abstract}

\section{THE ANIMAL SPECIES INTRODUCED INTO THE CORSICAN FRESHWATERS.}

\begin{abstract}
Seventeen new animal species, among which fifteen fish species, were introduced into the island within less than one hundred years. These species adapted to various aquatic environments, thus greatly contributing to the biodiversity of the fauna of the island, and especially to the fish populations in natural and artificial lakes. We found that the introduction of the new fish species also propagated an Anguillicola disease in eels. Furthermore, recent studies on wild brown trout populations revealed a genetic impact of the introductions.
\end{abstract}

\section{INTRODUCTION}

La Corse a une faune originale, caractérisée par un endémisme important qui concerne des populations isolées sur l'île depuis un temps variable selon les espèces, dont certaines ont une origine qui remonte au tertiaire (GIUDICELLI, 1971). Elle héberge des espèces d'origines orientales et méridionales, parvenues lors des échanges génétiques plus ou moins fréquents qui se sont produits avec les régions limitrophes et plus particulièrement l'Italie.

Comme dans toute île, la faune est soumise à une compétition en vase clos, et le déclin des espèces qui ne parviennent pas à se maintenir n'est en général pas compensé par un apport d'espèces exogènes, sauf introduction volontaire. 


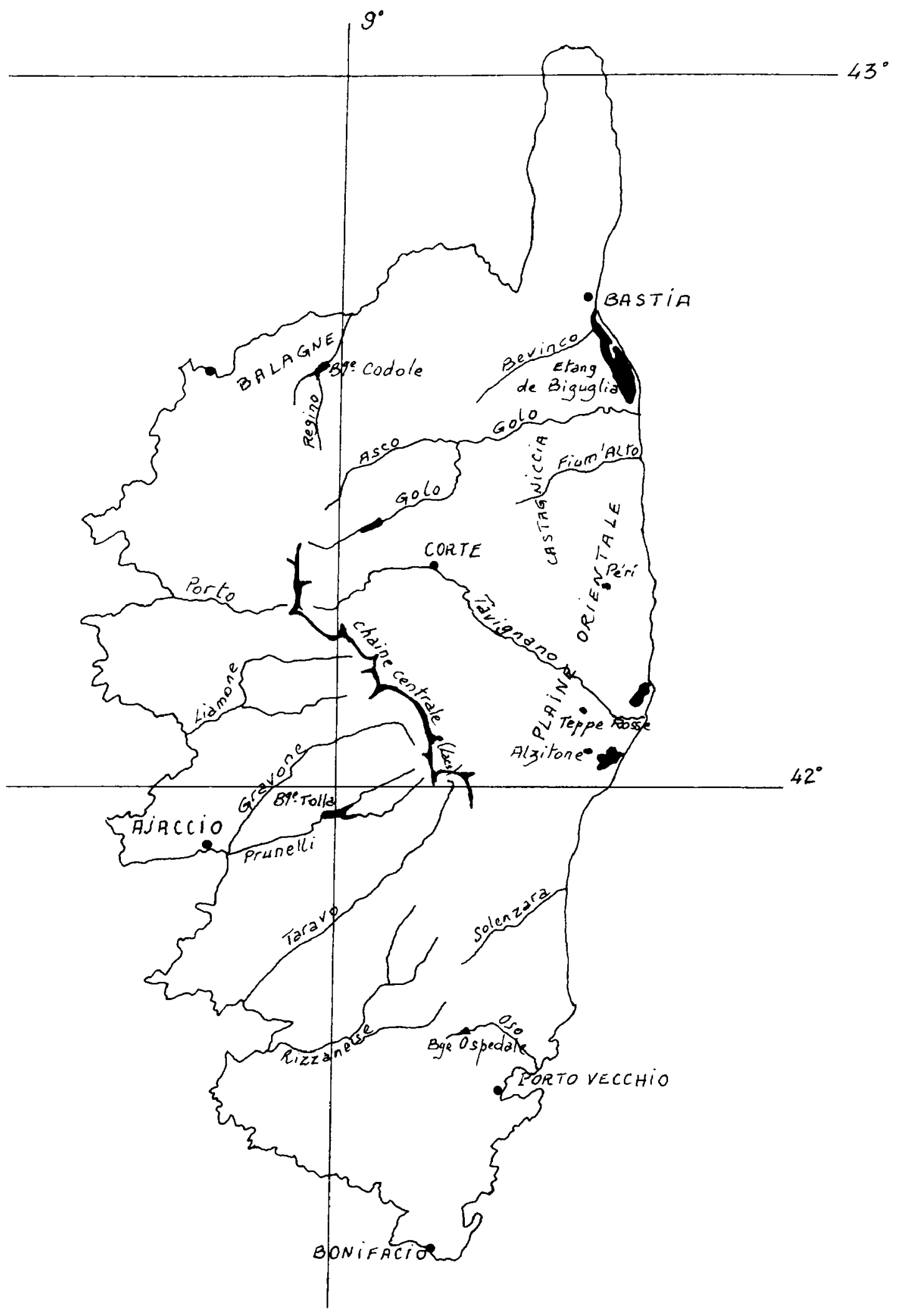

Figure 1

Réseau hydrographique de la Corse.

Figure 1

Drainage area of Corsica. 
Au cours des temps géologiques, la faune corse s'est donc appauvrie, les espèces insuffisamment compétitives ayant laissé la place aux espèces concurrentes les mieux adaptées. Ce phénomène est particulièrement marqué chez les poissons des eaux courantes, dont le peuplement autochtone est constitué seulement de quatre espèces dulçaquicoles (ROULE, 1933 ; SPILLMANN, 1961 ; GAUTHIER et ROCHE, 1974 ; ROCHE, 1987) : torrents ;

- la Truite, Salmo trutta macrostigma (Duméril, 1858), abondante dans tous les

- l'Anguille, Anguilla anguilla (Linné, 1758), très abondante en plaine et dans les cours d'eau littoraux, se rencontre jusqu'à $1000 \mathrm{~m}$ d'altitude ;

- la Blennie fluviatile, Blennius fluviatilis (Asso, 1801), présente dans les parties inférieures des grandes rivières;

- I'Epinoche, Gasterosteus aculeatus (Linné, 1758), très localisée et qui semble former des populations relictuelles peu abondantes.

Dans ce contexte, les espèces introduites doivent s'adapter aux contraintes du milieu torrentiel méditerranéen, mais bénéficient de conditions favorables lieees à l'absence d'espèces concurrentes et à l'existence de nombreuses niches écologiques vides.

Les premières introductions en Corse remontent à un siècle, et leur nombre s'est accru au cours de la dernière décennie. Au total, quinze espèces de poissons, une espèce de tortue et deux espèces d'écrevisses ont été introduites et contribuent à " l'enrichissement " de la biodiversité, tout en présentant des risques potentiels d'ordre écologique, sanitaire ou bien génétique.

\section{LES POISSONS}

\section{II.1. Les espèces introduites}

La plus ancienne introduction connue remonte à la fin du XIXème siècle et concerne la Gambusie, Gambusia affinis (Baird et Girard, 1853). Elle a été effectuée dans le cadre de la mise en oeuvre d'un vaste programme de lutte antipaludique, concernant l'assèchement par drainage des zones humides de la plaine orientale (BRUMPT, 1928). La Gambusie s'est acclimatée et développée dans le réseau de canaux de drainage et les parties inférieures des cours d'eau en communication avec ces canaux. D'autres secteurs littoraux, en relation avec les embouchures des principaux fleuves côtiers, ont également été concernés par cette introduction.

On peut considérer qu'en l'absence d'espèces concurrentes, il n'y a pas eu d'impact écologique majeur sur les peuplements en place, ni de compétition avec les populations d'Aphanius de Corse, Aphanius fasciatus (Cuvier et Valenciennes, 1821), petit Cyprinodonte euryhalin des eaux saumâtres qui occupe une niche écologique différente (VIDAL, 1995).

En revanche, en l'absence de prédateurs piscicoles, les populations de Gambusie peuvent connaitre localement des développements importants (cas du barrage de Codole sur le Regino en Balagne).

En 1970, des introductions ont été réalisées pour peupler, d'une part, certains lacs d'altitude et, d'autre part, les retenues collinaires créées dans le cadre de la mise en valeur agricole de la plaine orientale.

- La première opération, réalisée conjointement par la Fédération Interdépartementale pour la Pêche et la Protection des Milieux Aquatiques et le Parc Naturel Régional, concernait huit lacs naturels répartis entre 1700 et $2300 \mathrm{~m}$ d'altitude, dont certains étaient vierges de tout peuplement piscicole et d'autres abritaient des populations de truites autochtones. 
Elle avait pour objectif d'introduire la Truite fario d'élevage, Salmo trutta (Linné, 1758) et l'Omble de fontaine, Salvelinus fontinalis (Mitchill, 1815).

Les études piscicoles réalisées à partir des années 80 sur sept de ces lacs ont montré un échec total de l'opération dans trois cas : lacs de Goria, du Rotondo et de Bracca, et le déclin des populations introduites pour le lac de Capitello (GAUTHIER et al., 1984). Dans deux cas seulement, les populations d'ombles de fontaine se sont remarquablement bien acclimatées dans les lacs de Melo et de Bastani, et ont contribué à la disparition, dans le lac de Melo, de la population de truites autochtones (RIVIER, 1996 ; RIVIER et DUMONT, 1987, 1988, 1996a, 1996b).

L'omble de fontaine a migré dans les émissaires et les petits ruisseaux communiquant avec les lacs, où il forme des populations pérennes très bien adaptées à ces biotopes. II n'y a pas eu d'acclimatation simultanée de la truite et de l'omble de fontaine dans un même lac.

Au niveau de l'impact sur la faune autochtone, on a pu constater, à l'analyse de contenus stomacaux, que ce salmonidé exerçait une prédation sur une espèce d'urodèle endémique : l'euprocte, Euproctus montanus (Savi, 1838), dont les populations pourraient à terme disparaître de ces lacs.

- La seconde opération d'introduction avait pour objectif de créer une deuxième catégorie piscicole inexistante naturellement dans l'île, en peuplant trois plans d'eau artificiels de la plaine orientale de l'île n'ayant aucune communication avec le réseau hydrographique. Les espèces suivantes ont été introduites dans les réservoirs de Peri, Teppe-Rosse, et Alzitone :

le Gardon, Rutilus rutilus (Linné, 1758);

le Rotengle, Scardinius erythrophtalmus (Linné, 1758);

la Tanche, Tinca tinca (Linné, 1758);

la Carpe, Cyprinus carpio (Linné, 1758) et sa forme dépourvue d'écailles, la Carpe cuir ;

le Sandre, Stizostedion lucioperca (Linné, 1758).

Ces espèces ont ensuite été disséminées par les pêcheurs, dans divers milieux de l'île : barrages (Ospedale, Tolla), gravières abandonnées (Porto-Vecchio) ou en exploitation (Gravona), canaux (Biguglia), et cours inférieurs de grandes rivières (Gravona, Tavignano).

D'autres espèces ont été introduites par la suite, en dehors de tout programme organisé :

le Carassin doré, Carassius auratus (Linné, 1758) ;

le Carassin, Carassius carassius (Linné, 1758) ;

la Perche, Perca fluviatilis (Linné, 1758);

le Brochet, Esox lucius (Linné, 1758);

le Goujon, Gobio gobio (Linné, 1758) ;

le Poisson-chat, Ictalurus melas (Rafinesque, 1820);

et la Grémille, Gymnocephalus cernua (Linné, 1758).

De profondes modifications sont d'ores et déjà apparues dans la composition des peuplements piscicoles de certains barrages et cours inférieurs de rivières.

Les barrages de l'Ospedale et de Tolla ont des peuplements plurispécifiques de deuxième catégorie, avec pour le second la présence de neuf espèces. Dans ce barrage, la truite a disparu et l'eutrophisation du plan d'eau a favorisé la prolifération du gardon et de la perche (RIVIER et MOLINA, 1995). 
Dans le cours inférieur des grands fleuves remodelés et artificialisés par les extractions d'alluvions, certaines espèces comme le gardon, le goujon, le rotengle, le carassin et la perche sur la Gravona, ou le poisson-chat sur le Golo, prospèrent et forment des populations pérennes. Ces espèces trouvent abri dans ces milieux transformés, même en période de crues torrentielles, alors qu'elles ont peu de chances de survie dans les milieux naturels.

Enfin, il faut signaler les déversements réguliers de truite arc-en-ciel, Oncorhynchus mykiss (Walbaum, 1792) et de truite fario d'élevage, Salmo trutta fario (Linné, 1758) mis en oeuvre chaque année par les associations agréées de pêche pour le repeuplement des rivières et le développement de la pêche amateur.

\section{II.2. L'impact sanitaire}

En l'absence de tout contrôle sanitaire, l'introduction d'espèces constitue un mode d'apport potentiel de maladies et de parasites nouveaux, qui représentent autant de menaces pour la faune autochtone. Dans ce domaine, l'absence d'études ne permet pas d'établir de bilan, mais des observations récentes semblent confirmer ce risque.

Ainsi, l'apparition récente de l'anguillicolose dans la Gravone (ROCHE, 1994), parasitose due au nématode Anguillicola crassus (Kuwhara, Niimi et Igaki, 1974), semble être à l'origine de mortalité chez l'anguille. Sa présence a été mise en relation avec l'introduction du goujon, qui serait un hôte paraténique des larves du parasite (BLANC, comm. pers.).

Une autre espèce d'anguillicolose non identifiée a été trouvée dans l'étang de Biguglia. Elle pourrait avoir une origine italienne, et son introduction serait consécutive à l'apport d'anguilles de repeuplement. Ainsi, les baisses de production enregistrées sur cet étang, imputées jusqu'alors à la pollution du plan d'eau, trouveraient là une explication complémentaire.

\section{II.3. L'impact génétique}

II est actuellement bien connu chez les populations de truites sauvages qui sont constituées de deux souches génétiquement bien différenciées (BERREBI, 1995); la première est de type méditerranéen, la seconde est particulière à la Corse et serait la truite Salmo trutta macrostigma (Duméril, 1858) (KRIEG et GUYOMARD, 1983 ; BERREBI, 1995). Ces populations forment un patrimoine génétique original, plus ou moins menacé par les introductions de truites d'élevage dont la souche est d'origine atlantique. L'impact se traduit par un degré d'introgression qui affecte l'ensemble des populations naturelles, mais dont l'intensité, à quelques exceptions près, reste globalement faible, ce qui semble indiquer que la situation n'est pas irréversible, et que tout arrêt d'introductions nouvelles de truites d'élevage permettrait aux souches sauvages d'éliminer les gènes domestiques introduits.

\section{LES REPTILES}

L'apparition récente de la tortue de Floride (Trachemys scripta) dans divers milieux (plans d'eau, zones humides, rivières) indique que cette espèce élevée et commercialisée en aquariophilie est, comme partout ailleurs, relâchée dans le milieu naturel. A terme, elle peut présenter, si son acclimatation probable est effective, une menace pour la cistude d'Europe, Emys orbicularis (Linné, 1758), très abondante en Corse, avec laquelle elle se trouvera en compétition directe (DELAUGERRE et RECORBET, comm. pers.).

\section{LES INVERTÉBRÉS}

L'acclimatation de l'écrevisse à pieds blancs, Austropotamobius pallipes pallipes (Lereboullet, 1758), dans certains ruisseaux de Castagniccia est considérée comme une réussite intéressante en l'absence d'espèces d'écrevisses autochtones (ATTARD et VIANET, 1985). 
Les populations sont abondantes, indemnes de toutes maladies et parasitoses (ARRIGNON et ROCHE, 1983; ARRIGNON et al., 1988), et la protection des milieux est programmée par la Fédération de la pêche afin de constituer un sanctuaire national pour l'espèce.

Cependant, l'introduction récente de l'écrevisse américaine, Orconectes limosus (Rafinesque, 1817), couramment commercialisée vivante malgré la réglementation en vigueur, et acclimatée dans les gravières de la Gravona, pourrait favoriser l'importation de maladies et de parasitoses jusqu'alors inconnues. Une compétition entre les deux espèces n'est pas à exclure et pourrait constituer un danger pour les populations de Castagniccia si la dissémination de l'écrevisse américaine était poursuivie.

\section{CONCLUSION}

Quinze espèces de poissons, deux espèces d'écrevisses et une espèce de tortue aquatique ont été introduites dans les eaux douces de Corse, au cours du vingtième siècle.

Ces introductions, programmées dans un premier temps avec des objectifs définis, sont depuis une quinzaine d'années, la plupart du temps, le résultat d'actions individuelles non contrôlées. Elles constituent un phénomène préoccupant dont l'ampleur tend à augmenter et qui présente des risques d'ordre écologique, sanitaire ou génétique qui ne doivent pas être négligés.

En l'absence d'actions réglementaires efficaces, il conviendrait de mettre en oeuvre une information et une sensibilisation très large du public. Dans ce domaine, et plus particulièrement dans le cadre de la protection des souches sauvages de truites, un programme d'action est actuellement mis en place pour permettre la sauvegarde des populations naturelles. $\mathrm{Ce}$ programme est mené en partenariat avec la Fédération Interdépartementale pour la Pêche et les Milieux Aquatiques, le Parc Naturel Régional, la Collectivité Territoriale de Corse, l'Office de l'Environnement et la Direction Régionale de l'Environnement.

\section{BIBLIOGRAPHIE}

ARRIGNON J., ROCHE B., 1983. Population of the crayfish Austropotamobius pallipes pallipes Lereb. in a brook of Corsica (France). In Freshwater crayfish -V- Avi Goldman, Westport Connecticut, 229-238.

ARRIGNON J., MAGNE P., ATTARD J., ROCHE B., NICOD C., 1988. Dynamics of crayfishes populations, Austropotamobius pallipes pallipes Lereb. Verh. Internat. Verein. Limnol., 23, 2114-2117.

ATTARD J., VIANET R., 1985. Variabilité génétique et morphologique de cinq populations de l'écrevisse européenne Austropotamobius pallipes Lereb., 1858. (Crustacea, Decapoda). Can. J. Zool., 63 (12), 2933-2939.

BERREBI P., 1995. Etude génétique des truites de Corse. Rapp. final Parc Naturel Régional de Corse, 1-36.

BRUMPT E., 1928. Rôle du poisson vivipare américain Gambusia holbrooki dans la lutte contre le paludisme en Corse. C. R. Acad. Sciences, CLXXXVI, 909.

GAUTHIER A., ROCHE B., 1974. Présence de l'épinoche en Corse. Bull. Soc. Sc. Hist. Nat. Corse, 613, 41-48.

GAUTHIER A., ROCHE B., FRISONI G.F., 1984. Contribution à la connaissance des lacs d'altitude de la Corse. Parc Naturel Régional de Corse, $220 \mathrm{p}$.

GIUDICELLI J., 1971. Analyse de l'endémisme dans la faune des eaux courantes de la Corse. Ecologia Mediterranea, 1, 133-147. 
KRIEG F., GUYOMARD R., 1983. Mise en évidence électrophorétique d'une forte différenciation génétique entre populations de truite fario de Corse. C. R. Acad. Sci. Paris, 296, 10891094.

RIVIER B., 1996. Compte rendu des inventaires ichtyologiques du lac de Rotondo (Haute Corse) 1986-1987. Rapp. Cemagref, Parc Naturel Régional de Corse, 9 p.

RIVIER B., DUMONT B., 1987. Etude ichtyologique des lacs d'altitude de la Corse : le lac de Bastani. Cemagref, Parc Naturel Régional de Corse, $50 \mathrm{p}$.

RIVIER B., DUMONT B., 1988. Etude ichtyologique des lacs d'altitude de la Corse : le lac du Rotondo, le lac de Nino. Cemagref, Parc Naturel Régional de Corse, 91 p.

RIVIER B., MOLINA A., 1995. Etude du peuplement ichtyologique de la retenue de Tolla (Corse du Sud), éléments de gestion piscicole. Rapp. Cemagref, Fédération Interdépartementale pour la Pêche et la Protection des Milieux Aquatiques de la Corse, $16 \mathrm{p}$.

RIVIER B., DUMONT B., 1996a. Compte rendu de l'inventaire ichtyologique du lac de Bracca, campagne de 1989. Rapp. Cemagref, Parc Naturel Régional de Corse, 8 p.

RIVIER B., DUMONT B., 1996b. Compte rendu de l'inventaire ichtyologique du lac de Vitalaca, campagne de 1989. Rapp. Cemagref, Parc Naturel Régional de Corse, $15 \mathrm{p}$.

ROCHE B., 1987. Poissons dulçaquicoles de la Corse. Etude du peuplement piscicole des eaux courantes et des populations de truites. Rapp. SRAE Minist. Agric., 37 p.

ROCHE B., 1994. Etude piscicole du cours inférieur de la Gravone (Corse du sud). Rapp. DIREN Minist. Envir., $13 \mathrm{p}$.

ROULE L., 1933. Le peuplement des cours d'eau de la Corse en poissons. Bull. Fr. Piscic., 63, 61-62.

SPILLMANN C.J., 1961. Poissons des eaux douces de la Corse. In LECHEVALLIER (ed.), Poissons d'eau douce. Faune de France, 65, 224-230.

VIDAL S., 1995. Biologie, biométrie et écologie d'un poisson des eaux littorales de Corse : Aphanius fasciatus Nardo, 1827. (Teléostéen, Cyprinodontidae). Mém. DESS Univ. Corse, $45 \mathrm{p}$. 